\title{
Negative Magnetoresistance in the Variable-Range-Hopping Transport of Strongly Underdoped $\mathrm{La}_{2-x} \mathrm{Sr}_{x} \mathrm{CuO}_{4}$
}

\author{
A. Malinowski ${ }^{a}$, M.Z. Cieplak $^{a}$, M. Berkowski $^{a}$ \\ AND S. GUHA ${ }^{b}$ \\ ${ }^{a}$ Institute of Physics, Polish Academy of Sciences \\ al. Lotników 32/46, 02-668 Warsaw, Poland \\ ${ }^{b}$ Department of Physics and Astronomy, Rutgers University \\ Piscataway, NJ 08885, USA
}

\begin{abstract}
The in-plane transport of strongly underdoped $\mathrm{La}_{2-x} \mathrm{Sr}_{x} \mathrm{CuO}_{4}$ films was examined in the magnetic fields up to $14 \mathrm{~T}$ and in temperatures down to 1.6 K. While at high temperatures the samples display metallic-like resistivity, the low- $T$ transport is governed by variable-range-hopping mechanism. Careful analysis shows that the temperature dependence of pre-exponential factor in Mott's variable-range-hopping law may not be neglected and that the density of states at the Fermi level can be effectively expressed as $g\left(E-E_{\mathrm{F}}\right)=N_{0}\left(E-E_{\mathrm{F}}\right)^{p}$, with a small exponent $p$ of the order of 0.1. In the magnetic field parallel to $\mathrm{CuO}_{2}$ planes one of the variable-range-hopping parameter, $\rho_{0}$, increases by about $20-25 \%$, while the other one, $T_{0}$, decreases by about $10-15 \%$, resulting in the decrease in total resistivity. This effect may be related to the decrease of the tunneling barrier between different antiferromagnetic clusters in the presence of magnetic field.
\end{abstract}

PACS numbers: 74.72.--h, 74.25.Fy, 73.43.Qt

\section{Introduction}

The evolution of the electronic structure in high- $T_{\mathrm{C}}$ cuprates (HTSC), from the charge-transfer type of the Mott insulator through spin-glass phase to superconductor, is still one of these intriguing issues which are believed to lay in the center of HTSC physics. Obviously, doping-induced changes in the character of electronic states affect strongly macroscopic properties, including transport and magnetic ones. The metallic-like behavior (i.e. positive slope $\mathrm{d} \rho / \mathrm{d} T$ ) is observed in the transport at high temperatures even deeply inside the antiferromagnetic 
phase $(x<0.02)$ (which otherwise clearly displays an insulating character at lower $T$, where transport is governed by variable-range-hopping (VRH) mechanism) and even in these specimens where the $k_{\mathrm{F}} l$ parameter $\left(k_{\mathrm{F}}\right.$ is the Fermi wave vector and $l$ is the mean-free path) estimated from the resistivity is far below the Mott limit for metallic resistivity in two-dimensional metal. This has not been understood until very recently. The angle-resolved photoemission (ARPES) measurements [1] reveals a rich and unusual electronic structure of underdoped $\mathrm{La}_{2-x} \mathrm{Sr}_{x} \mathrm{CuO}_{4}$ (LSCO), with two dominated features: the "arc" of quasi-particles (QP) formed at Fermi surface by the states in the nodal direction of the $d$-wave superconductor, $(0,0)-(\pi, \pi)$, and a broad feature around $(\pi, 0)$ located $0.2 \mathrm{eV}$ below the Fermi level. The existence of QP arc, already for as small hole doping as $x=0.03$, can explain metallic-like high-temperature transport in specimens where otherwise a VRH transport in low temperatures is observed. The states from the gapped region around $(\pi, 0)$, considered to be related to stripes [2], do not participate in metallic transport but can take part in transport via a VRH mechanism. In the light of above, however, an attempt to describe VRH in underdoped LSCO by the familiar formula: $\rho(T)=\rho_{0} \exp \left(T_{0} / T\right)^{1 / 4}$, i.e. neglecting temperature dependence of pre-exponential factor and assuming constant density of states in the vicinity of the Fermi level, $E_{\mathrm{F}}$, seems to be too-far going oversimplification. While this can give a reasonable fit to experimental data, this may not be in agreement with theoretical predictions [3].

The metallic transport at high $T$ was shown to be insensitive to the onset of long-range antiferromagnetic $(\mathrm{AF})$ order, suggesting that the doped carriers are decoupled from the background spins in $\mathrm{CuO}_{2}$ planes [4]. On the other hand, it is well known that small hole doping $(x=0.02)$ is sufficient to destroy the Néel ordering, indicating a strong coupling of these two subsystems [5]. Magnetoresistance measurements may be a useful probe to shed some light onto this puzzle.

In the spin-glass phase of LSCO, between high- $T$ metallic conduction and a low- $T$ regime of the $\mathrm{VRH}$, there is an intermediate- $T$ transition region where conductivity is proportional to $\ln T$. Recently, we clarify that this logarithmic $T$-dependence is caused by a strong electron-electron (e-e) interaction, rather than by conventional weak localization in two-dimensional system [6]. If so, e-e interactions should be also important at low- $T$ and influence on the VRH-law parameters.

\section{Results and discussion}

We study the in-plane resistivity of strongly underdoped LSCO films, with $x=0.03,0.045,0.048$, in the magnetic fields up to $14 \mathrm{~T}$, applied parallel to $\mathrm{CuO}_{2}$ planes, and in temperatures down to $1.6 \mathrm{~K}$. Zero-field temperature dependence of resistivity below $20 \mathrm{~K}$ for all samples is shown in Fig. 1. The derivative of $\ln \rho$ over $T^{-1 / 4}$, calculated for the sample with $x=0.03$, i.e. where VRH range extends up to the highest $T$, is depicted in the inset to Fig. 1 as a function of $T^{-1 / 4}$. The 
derivative increases by almost $40 \%$ between 0.47 and 0.89 (i.e. between $20 \mathrm{~K}$ and $1.6 \mathrm{~K}$ ) and shows no sign of saturation. This indicates strong $T$-dependence of the prefactor in Mott's VRH law. To take into account the variation of the density of states in the vicinity of $E_{\mathrm{F}}, g\left(E-E_{\mathrm{F}}\right)$, we fit $\rho(T)$ data to the following formula:

$$
\rho(T)=\rho_{0} T^{2 w} \exp \left(\left(T_{0} / T\right)^{w}\right) .
$$

This formula was obtained for the assumption that $g\left(E-E_{\mathrm{F}}\right)=N_{0}\left(E-E_{\mathrm{F}}\right)^{p}$ and $w=(p+1) /(p+4)$ [7]. We applied it to $\rho(T)$ data below the lower limit of $\sigma \sim \ln T$ dependence, $T_{\ln }$, equal to $20 \mathrm{~K}, 15 \mathrm{~K}$, and $12 \mathrm{~K}$ for $x=0.03,0.045$, and 0.048, respectively. Best fits, shown in Fig. 1 as solid lines, are found for $p=0.21$ (i.e. $w=0.29), 0.04(w=0.26)$, and $0.08(w=0.26)$ (for $x=0.03$, $0.045,0.048$, respectively). The values of other fitting parameters are given in the caption to Fig. 1 . The $T_{0}$, being inversely proportional to $a^{3}$ ( $a$ - the localization

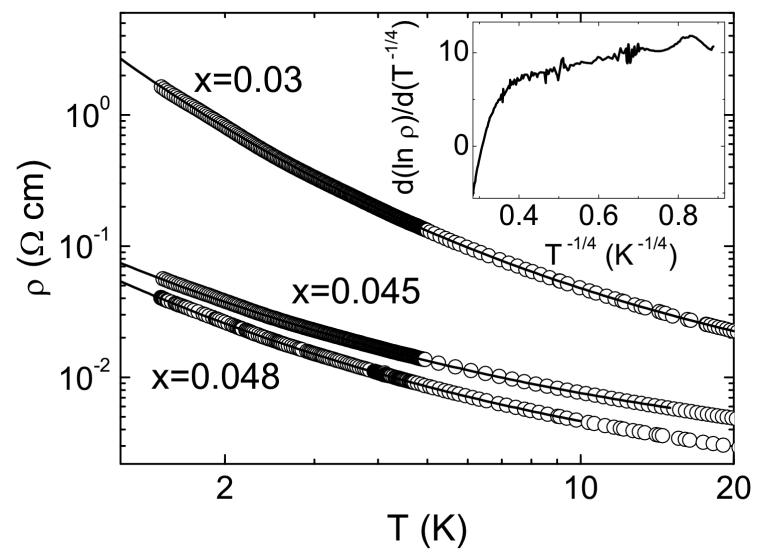

Fig. 1. Resistivity of underdoped LSCO below $20 \mathrm{~K}$ for different $x$. Solid lines show the best fits to Eq. (1), which were found for $T_{0}=6.17 \times 10^{3}\left(4.09 \times 10^{3}\right.$ and $\left.3.67 \times 10^{3}\right) \mathrm{K}$ and $\rho_{0}=2.19 \times 10^{-5}\left(2.06 \times 10^{-5}\right.$ and $\left.1.11 \times 10^{-5}\right) \Omega \mathrm{cm}$ for $x=0.03$ (0.045 and 0.048$)$, respectively. The inset shows derivative of $\ln \rho$ over $T^{-1 / 4}$.

length), increases as $x$ approaches the metal-to-nonmetal transition at $x \approx 0.02$. The character of $\rho(T)$ dependence for $x=0.03$ does not change with temperature - reducing the upper limit of the fitted $T$-range to $(1 / 2) T_{\ln }$ changes the obtained parameters not more than $10 \%$. For $x=0.045$ and $x=0.048$, however, this results in increasing $p$ up to 0.17 and 0.14 , respectively. The exponent $p$ describes effective (averaged for all wave vectors $k$ ) modification of the density of states in the vicinity of the Fermi level caused by the presence of e-e interactions. The effective $p$ just below $T_{\ln }$ is essentially equal to 0 for $x=0.045$ and $x=0.048$ which corresponds to Mott's expression for VRH in the absence of e-e correlations. Apparently, the carrier concentration in samples with $x \geq 0.045$ is sufficiently high to provide screening of e-e interactions. The screening become weaker at lower $T$, as suggested by the increase in $p$ with decreasing $T$. A larger variation of $g\left(E-E_{\mathrm{F}}\right)$ 
for $x=0.03$, manifested by larger $p$, indicates that the hole concentration is not high enough to provide an adequate screening of e-e interaction. In addition, it may be related to the aforementioned broad feature observed in ARPES spectra around $(\pi, 0)$ and believed to be connected to stripes [1, 2].

Magnetic field applied parallel to $\mathrm{CuO}_{2}$ planes retains VRH-like character of in-plane transport, albeit changes both parameters, $\rho_{0}$ and $T_{0}$. The value of $\rho_{0}$ increases by about $20-25 \%$ at $14 \mathrm{~T}$ with respect to its zero-field value and $T_{0}$ decreases by about 10-15\% (see Fig. 2). The combined effect of both changes is the decrease in resistivity, because the negative change of the exponential term in Eq. (1) overcomes the positive change of $\rho_{0}$. The magnitude of the total effect
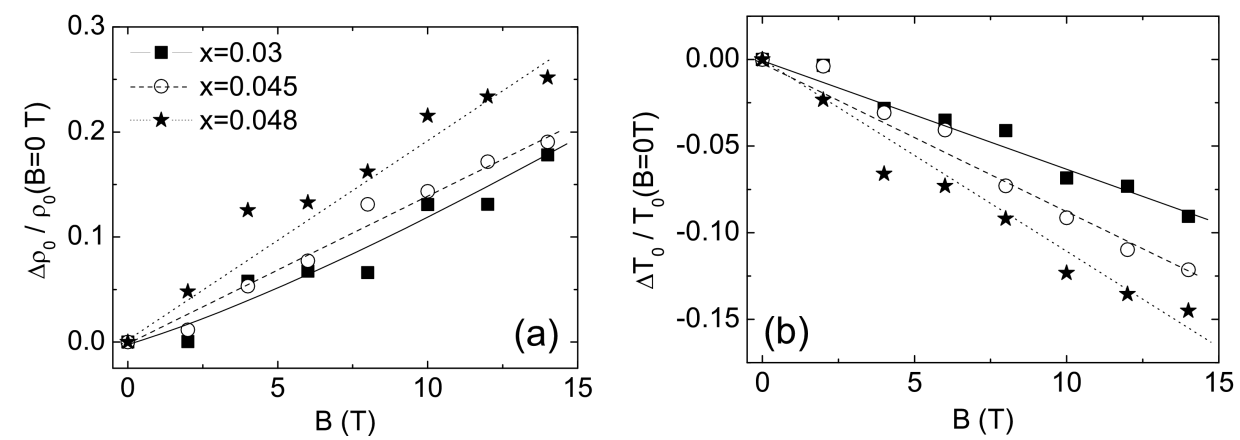

Fig. 2. The field-induced change of the VRH law parameters: (a) $\rho_{0}$ and (b) $T_{0}$ for all three samples. The lines are guides for eyes.

(i.e. decreasing of resistivity) is the biggest for $x=0.03$. In the original VRH theory both $\rho_{0}$ and $T_{0}$ are inversely proportional to the density of state, so the opposite way in which $\rho_{0}$ and $T_{0}$ change with field is not easy to interpret. Some of the recently modified VRH theories, trying to describe magnetoresistance in the paramagnetic phase of manganites [8], introduce a field-dependent hopping barrier (to which $T_{0}$ is related) or a term in $\rho_{0}$ inversely proportional to the square of magnetization. There are also suggestions that transport in manganites is a process involving a combination of hopping and tunneling between magnetic clusters. It is possible that a similar situation takes place in underdoped LSCO. The carriers are believed to be confined to the regions between antiferromagnetically oriented clusters of spins. Thus, both hopping inside hole-rich areas and hopping and tunneling between different clusters may be involved in the transport. Applied magnetic field tends to decrease the tunneling barrier and enhance the conductivity. This scenario needs a theoretical evaluation. Our experimental results showing opposing changes of both hopping parameters, $\rho_{0}$ and $T_{0}$, offers a good testing background for such theoretical models of VRH transport in insulating LSCO. 
Negative Magnetoresistance in the Variable-Range-Hopping Transport ...615

\section{Acknowledgment}

This work was supported by the State Committee for Scientific Research (KBN) grant P2 P03B 04423.

\section{References}

[1] A. Ino, C. Kim, M. Nakamura, T. Yoshida, T. Mizokawa, Z.-X. Shen, A. Fujimori, T. Kakeshita, H. Eisaki, S. Uchida, Phys. Rev. B 62, 4137 (2000); T. Yoshida, X.J. Zhou, T. Sasagawa, W.L. Yang, P.V. Bogdanov, A. Lanzara, Z. Hussain, T. Mizokawa, A. Fujimori, H. Eisaki, Z.-X. Shen, T. Kakeshita, S. Uchida, Phys. Rev. Lett. 91, 027001 (2003).

[2] X.J. Zhou, T. Yoshida, S.A. Kellar, P. V. Bogdanov, E.D. Lu, A. Lanzara, M. Nakamura, T. Noda, T. Kakeshita, H. Eisaki, S. Uchida, A. Fujimori, Z. Hussain, Z.-X. Shen, Phys. Rev. Lett. 86, 5578 (2001).

[3] T.G. Castner, Phys. Rev. B 61, 16596 (2000).

[4] Y. Ando, A.N. Lavrow, S. Komiya, K. Segawa, X.F. Sun, Phys. Rev. Lett. 87, 017001 (2000).

[5] M.A. Kastner, R.J. Birgeneau, G. Shirane, Y. Endoh, Rev. Mod. Phys. 70, 897 (1998).

[6] Marta Z. Cieplak, A. Malinowski, S. Guha, M. Berkowski, Phys. Rev. Lett. 92, 187003 (2004).

[7] F.D. Allen, C.J. Adkins, Philos. Mag. 26, 1027 (1972).

[8] P. Wagner, I. Gordon, L. Trappeniers, J. Vanacken, F. Herlach, V.V. Moshchalkov, Y. Bruynseraede, Phys. Rev. Lett. 84, 4018 (2000) and references citied. 